QUANTITATIVE GENETICS

\section{Turning up the heat on QTL mapping}

Yeast may be smaller and less complicated than other eukaryotes, but don't count on them for having simpler genetics. This is well exemplified in a recent report in Nature by Steinmetz et al., who examined a quantitative trait that yields a high-temperature growth $(\mathrm{Htg})$ phenotype in selected Saccharomyces cerevisiae isolates. The unexpected complexity of their results serves as a warning for mappers of quantitative trait loci (QTL) in other organisms.

In order to map the Htg trait, Steinmetz et al. crossed two strains of S. cerevisiae: one strain with the Htg trait and one strain without it. They then used microarray analysis to compare the entire genomes of, and to map recombination points in $\mathrm{Htg}^{+}$and $\mathrm{Htg}^{-}$progeny of this cross, allowing them to pinpoint a locus on chromosome XIV that appeared to be linked to the Htg trait. However, neither the extensive sequencing of this locus nor the comparison of gene expression levels in this region provided a clear answer as to what gene - or genes - underlie this mapped QTL.

So to overcome this problem, Steinmetz et al. used a novel strategy called reciprocal hemizygosity analysis, in which new diploid strains were created that contained a haploid deletion in one or the other parental chromosome at a single gene of interest. In this way, each allele of a gene could be examined separately for its individual contribution to the phenotype. For the chromosome XIV QTL, alleles of two genes, MKT1 and RHO2, from the $\mathrm{Htg}^{+}$parent contributed to the Htg phenotype in the progeny. But, surprisingly, one allele of a third gene, $E N D 3$, from the $\mathrm{Htg}$ parent also contributed to the $\mathrm{Htg}$ phenotype - a finding that helps to explain why the initial cross between the two strains produced a hybrid with heterosis (or 'hybrid vigour').

Such interesting results from a 'simple' organism might have unwelcome implications for attempts to map QTL in more complicated organisms - such as in agricultural crops, mice and humans - in which most QTL mapping is now taking place. The authors suggest that current techniques are likely to miss QTL if their effects are too small or if they are produced in part by unexpected combinations of alleles between strains, as with the Htg phenotype. Chris Gunter Associate Editor, Nature

\section{(2) References and links} ORIGINAL RESEARCH PAPER Steinmetz, L. M. et $a$. Dissecting the complex architecture of a quantitative trait locus in yeast. Nature 21 March 2002 (DOl 10.1038/nature732)

FURTHER READING Mackay, T. F. C.

Quantitative trait loci in Drosophila. Nature Rev Genet. 1, 11-20 (2001) | Barton, N. H. \& Keightley, $P$ P. D. Understanding quantitative genetic variation. Nature Rev. Genet. 2, 11-21 (2002) WEB SITE

Ron Davis' lab:

hittp://cmgm.stanford.edu/biochem/davis.htm

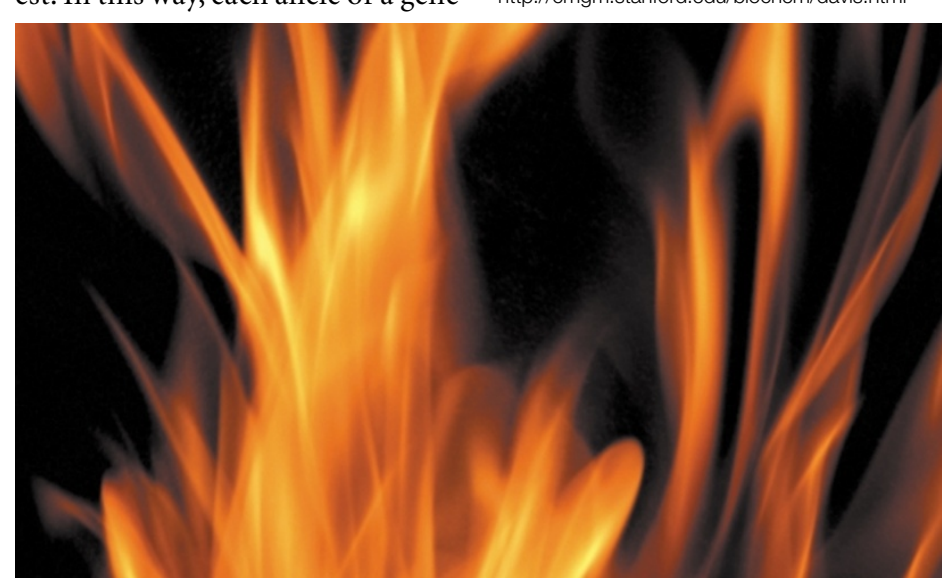

\section{IN BRIEF}

\section{EPIGENETICS}

Higher-order structure in pericentric heterochromatin involves a distinct pattern of histone modification and an RNA component.

Maison, C. et al. Nature Genet. 30, 329-334 (2002)

The non-coding Air RNA is required for silencing autosomal imprinted genes.

Sleutels, F. et al. Nature 415, 810-813 (2002)

Epigenetic regulation of gene expression is mainly achieved through modulation of chromatin states. Centromeric heterochromatin is essential for centromere function in yeast and mammals and accounts for transcriptional silencing. By selectively blocking hypoacetylation and methylation of histones in the mammalian centromeric region, Maison and colleagues found that these two types of modification can occur independently, on different histones. They also found that the association of an RNA molecule with the methylated histones is required for the accumulation of HP1 protein in this complex, which leads to stabilization of heterochromatin. The second paper, by Sleutels et al., reports that a non-coding RNA molecule is also involved in imprinting. The Air RNA is required for silencing of the Igf2r/Slc22a2/Slc22a3 gene cluster on the paternal chromosome - repression of this cluster correlates with Air RNA expression.

\section{BIOINFORMATICS}

Computational detection and location of transcriptional start sites in mammalian genomic DNA.

Down, T. A. et al. Genome Res.12, 458-461 (2002)

The promoters of complex species frequently contain several transcription start sites (TSSs), which are difficult to detect computationally because of the diffuse, complex structure of eukaryotic promoters. Here, Down and Hubbard describe a new bioinformatics program, Eponine, which they use in a novel approach to detect TSSs. They report that a TATA box in combination with $(\mathrm{C}+\mathrm{G})$-rich flanking sequence - and not a TATA box alone - gives the best indication of the presence of a TSS.

\section{HUMAN GENETICS}

A genome-wide linkage screen for relative hand skill in sibling pairs.

Francks, C. et al. Am. J. Hum. Genet. 70, 800-805 (2002)

This paper reports the first genome-wide screen for a measure that is related to handedness in humans and the identification of a putative quantitative trait locus (QTL) on chromosome 2p11.2-12 that influences relative hand skill in a sample of 195 dyslexic sibling pairs. However, Francks et al. found that this QTL was not significantly associated with relative hand skill in a second dyslexic sibling sample, indicating that this trait has a complex genetic aetiology. 